SCIENTIA AGRIPECUARIA

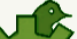

Facultad de Ciencias

Agropecuarias

Universidad Nacional de Trujillo

\title{
REVIEW
}

\section{Biopolymers produced by Azotobacter: synthesis and production, physico- mechanical properties, and potential industrial applications}

\author{
Biopolímeros producidos por Azotobacter: síntesis y producción, propiedades físico- \\ mecánicas, y potenciales aplicaciones industriales \\ Nils Leander Huamán-Castilla1,2, (DD); Erik Edwin Allcca-Alca ${ }^{1}$; ; Giovanna Judith Allcca-Alca3 (iD);
Marcos Luis Quispe-Pérez ${ }^{4}$ (D) \\ 1 Escuela de Ingeniería Agroindustrial, Universidad Nacional de Moquegua, Prolongación Calle Ancash s/n, Moquegua 18001. Peru. \\ 2 Instituto de Investigación para el Desarrollo del Perú (IINDEP), Laboratorio de Tecnologías Sustentables para la extracción de \\ Compuestos de Alto Valor, Universidad Nacional de Moquegua, Prolongación Calle Ancash s/n, Moquegua 18001. Peru. \\ 3 Escuela de Postgrado, Universidad Nacional del Altiplano, Av. Floral s/n, Puno 1153. Peru. \\ ${ }^{4}$ Escuela de Ingeniería de Minas, Universidad Nacional de Moquegua, Prolongación Calle Ancash s/n, Moquegua 18001. Peru.
}

*Corresponding author: nhuamanc@unam.edu.pe (N. L. Huamán-Castilla).

Received: 9 January 2021. Accepted: 30 May 2021. Published: 20 July 2021.

\begin{abstract}
Azotobacter is a type of bacterium nonpathogenic, Gram-negative with ability to synthesize simultaneously under aerobic conditions two biodegradable polymers extra and intracellular such as alginate and polyhydroxyalkanoates, respectively, whose physical, mechanical, and biodegradable properties have awoken the interest in the industry. Thus, this review compiles information on synthesis chemistry of these polymers and the genetic modification of this bacterium in order to produce a particular polymer and improve its yields. Additionally, we show that alginates obtained present a higher molecular weight, degree of acetylation and M/G ratio compared with other organic sources like seaweed; while polyhydroxyalkanoate polymers have better physical and mechanical characteristics (melting temperature, Young's modulus, elongation and glass transition temperature) compared with other synthetic polymers. These characteristics present in both polymers have allowed the development of several applications in the food, pharmaceutical, medical and agronomic industries. Finally, it is necessary to study new strategies to control the variability of the degree of acetylation and molecular weight for alginate polymers and improve the production of polyhydroxyalkanoates in order to propose new future applications in the industry.
\end{abstract}

Keywords: Azotobacter; polyhydroxyalkanoate (PHA); polyhydroxyvalerato (PHV); polyhydroxybutyrate (PHB); alginates (AG); polymers.

\begin{abstract}
Resumen
Azotobacter es un tipo de bacteria no patógena, Gram negativa con capacidad para sintetizar simultáneamente en condiciones aerobias dos polímeros biodegradables extra e intracelulares tales como alginatos y polihidroxialcanoatos respectivamente, cuyas propiedades físicas, mecánicas y biodegradables han despertado el interés en la industria. Por ello, esta revisión compila información sobre la síntesis química de estos polímeros y la modificación genética de esta bacteria con la finalidad de producir un polímero particular y mejorar sus rendimientos. Adicionalmente, mostramos que los alginatos obtenidos presentan un mayor peso molecular, grado de acetilación y relación M/G comparado con otras fuentes orgánicas como las algas marinas; mientras los polímeros de polihidroxialcanoatos presentan mejores características físicas y mecánicas (temperatura de fusión, módulo de Young, elongación y temperatura de transición vítrea) comparado con otros polímeros sintéticos. Estas características presentes en ambos polímeros han permitido el desarrollo de diversas aplicaciones en la industria alimentaria, farmacéutica, médica y agronómica. Finalmente, es necesario estudiar nuevas estrategias para controlar la variabilidad del grado de acetilación y peso molecular para los polímeros de alginato y mejorar la producción de polihidroxialcanoatos con la finalidad de promover nuevas futuras aplicaciones en la industria.
\end{abstract}

Palabras clave: Azotobacter; polihidroxialcanoatos; polihidroxibutirato (PHB); polihidroxivalerato (PHV); alginato (AG); polímeros.

DOI: https://dx.doi.org/10.17268/sci.agropecu.2021.040

Cite this article:

Huamán-Castilla, N. L., Allcca-Alca, E. E., Allcca-Alca, G. J., \& Quispe-Pérez, M. L. (2021). Biopolímeros producidos por Azotobacter: síntesis y producción, propiedades físico-mecánicas, y potenciales aplicaciones industriales. Scientia Agropecuaria, 12(3), 369-377. 


\section{Introducción}

La producción de plásticos sintéticos se ha incrementado considerablemente en el mundo ( 8300 millones de toneladas por año) (Geyer et al., 2017; Chamas et al., 2020). Estos plásticos sintéticos son en su mayoría polietileno, polipropileno y poliestireno, los cuales son usados como envases, embalajes, vehículos de transporte, equipos médicos e inclusive como materiales en prendas de vestir (Andrady \& Neal, 2009; Geyer et al., 2017). No obstante, estos materiales en particular no son biodegradables ocasionando problemas de gestión ambiental, con daños irreversibles al medio ambiente (Scott, 2015). El carácter hidrofóbico y la ausencia de grupos funcionales en la estructura química de estos plásticos sintéticos impide su degradación por bacterias, enzimas e inclusive por otros factores tales como la temperatura ambiente, luz y el agua (Rosato et al., 1991; Alshehrei, 2017). Por ejemplo, se estima que las bolsas de plástico pueden demorar en degradarse entre 10 a 20 años, mientras que las botellas de plástico pueden demorar entre 70 y 500 años (Urbanek et al., 2018; Chamas et al., 2020). Por ello, diversos trabajos se han enfocado en promover la producción y uso de polímeros biodegradables como una alternativa para reemplazar los plásticos sintéticos y reducir los problemas de gestión ambiental.

Los polímeros biodegradables son metabolitos secundarios producidos bajo condiciones de estrés (temperatura, presión, pH entro otros) a partir de microorganismos tales como hongos y bacterias (Mohapatra et al., 2017). Estos polímeros debido a su naturaleza hidrofílica y a la presencia de grupos funcionales en su estructura química pueden hidrolizarse fácilmente a monómeros y oligómeros a través de diferentes mecanismos de degradación biológica (Nair et al., 2016), cuyo periodo de degradación es relativamente corto entre 20 y 500 días (Volova et al., 2010). Por ello, la búsqueda de nuevas fuentes naturales alternativas capaces de producir biopolímeros a bajo costo no solo con la finalidad de reemplazar a los plásticos sintéticos, sino también que permitan reducir significativamente el daño al medio ambiente es aún una tarea pendiente por resolver.

Los polihidroxialcanoatos (PHAs) y el alginato (AG) son dos polímeros biodegradables obtenidos de diferentes fuentes naturales (algas marinas y microorganismos), los cuales han ido ganando popularidad debido a sus altos rendimientos y bajos costo de producción (Yoneyama et al., 2015). Si bien, estos polímeros son resistentes, flexibles, biocompatibles, no tóxicos y biodegradables; sus excelentes propiedades viscoelásticas, estabilizantes y gelificantes permiten diferentes aplicaciones en la industria médica, farmacológica y alimentaria (Francesca, 1997; Gautam et al., 2007; García et al., 2014). Consecuentemente, el uso de ambos biopolímeros permitiría sustituir a los plásticos sintéticos, contribuyendo al desarrollo sostenible de las industrias (Vieira et al., 2011).

Si bien diferentes estudios se han enfocado en determinar qué tipos de algas marinas y microorganismos pueden ser utilizadas como fuentes naturales para la producción de PHAs y AG (Rehm, 2010), son los microorganismos Gram positivos quienes presentan una serie de ventajas tales como su capacidad para sintetizar ambos polímeros simultáneamente y según la modificación genética pueden controlar el peso molecular de estos compuestos o en su efecto producir un solo polímero en particular (Galindo et al., 2007; Rehm, 2010). Azotobacter es un tipo de bacteria Gram positiva presente en suelo y agua dulce, que puede sintetizar simultáneamente PHAs y AG como mecanismo de protección natural, cuyas características químicas, físicas, mecánicas y termodinámicas de ambos polímeros han mostrado cualidades particulares que pueden ser utilizadas en futuras aplicaciones en la industria (Rehm, 2010; Yoneyama et al., 2015). Por ello, esta revisión compila información sobre la síntesis de estos polímeros a partir de las especies de Azotobacter, luego se discuten algunas de sus propiedades físicas y mecánicas; y finalmente se presenta su potencial uso como una nueva alternativa para el sector alimentario, medico, farmacéutico y agronómico.

\section{Género Azotobacter}

A la fecha han sido identificadas 7 especies del género Azotobacter, un grupo de bacterias Gram negativas, no patógenas, estrictamente aerobias con una gran habilidad de fijar $\mathrm{N}_{2}$ en el suelo y sintetizar sustancias que favorecen el crecimiento de las plantas tales como hormonas, vitaminas, aminoácidos y $\mathrm{N}_{2}$ (Choi \& Lee, 1999; Jiménez et al., 2011; Martyniuk \& Martyniuk, 2003; Sumbul et al., 2020). No obstante, cada una de estas especies tienen características particulares de crecimiento que las diferencian, así como también en algunos casos difieren en la producción de algunos metabolitos (Tabla 1).

Si bien este grupo de especies es estrictamente aerobio, bajo condiciones de estrés o condiciones ambientales desfavorables (baja concentración de oxígeno) tienen la capacidad de formar quistes para entrar en un estado de reposo o inactividad (Gauri et al., 2012). La creación de este tipo de barrera defensiva permite que este tipo de microorganismos pueda generar metabolitos intra y extracelulares tales como polihidroxialcanoatos (PHA), y alginatos (AG) (Tabla 1) (Patil et al., 2020).

\section{Síntesis y producción biopolímeros}

Si bien las diferentes especies de Azotobacter tienen la capacidad de producir los biopolímeros de AG y PHA, son finalmente las especies $A$. vinelandii y $A$. chroococcum las que mayor presencia tienen en los suelos fértiles (> 80\%) en comparación con las otras especies de Azotobacter (Aasfar et al., 2021). Por ello, el enfoque de los metabolitos extra e intracelulares producidos bajo condiciones de estrés por ambas especies de $A$. vinelandii y $A$. chroococcum son detallados a continuación.

\subsection{Alginato (AG)}

Bajo condiciones ambientales desfavorables, ambas especies de A. chroococcum y A. vinelandii tienen la capacidad de formar quistes con la finalidad de protegerse y entrar en un estado de reposo latente (Gawin et al., 2020). Estos quistes son capsulas compuestas por biopolímeros de AG, un tipo de metabolito extracelular, cuya estructura química presenta moléculas de ácido $\beta$-D-manurónico $(\mathrm{M})$, ácido $\alpha$-Lgulurónico $(G)$ y algunos grupos acetilados (O-acetilo) (Figura 1) (Castillo et al., 2013; Gawin et al., 2020). 
Tabla 1

Características morfológicas básicas del género Azotobacter

\begin{tabular}{|c|c|c|c|c|c|}
\hline Especie & $\begin{array}{l}\text { Tamaño de } \\
\text { célula }\end{array}$ & $\begin{array}{l}\text { Condiciones } \\
\text { normales de } \\
\text { crecimiento }\end{array}$ & $\begin{array}{c}\text { Metabolitos producidos } \\
\text { bajo condiciones normales } \\
\text { de crecimiento }\end{array}$ & $\begin{array}{c}\text { Biopolímeros producidos } \\
\text { bajo condiciones } \\
\text { controladas }\end{array}$ & Fuente \\
\hline $\begin{array}{l}\text { Azotobacter } \\
\text { chroococcum }\end{array}$ & $3,0-7,0 \mu \mathrm{m}$ & $\begin{array}{c}\mathrm{T}^{\circ}: 15-30^{\circ} \mathrm{C} \\
\mathrm{pH}: 7-7,4\end{array}$ & $\begin{array}{c}\text { Aminoácidos Vitamina B2 y } \\
\text { B3 } \\
\mathrm{CO}_{2} \\
\mathrm{NH}_{3} \\
\end{array}$ & $\begin{array}{l}\text { PHA } \\
\text { AG }\end{array}$ & $\begin{array}{l}\text { (Lenart, 2012) } \\
\text { (Chennappa et al., } \\
\text { 2019) } \\
\text { (Patil et al., 2020) }\end{array}$ \\
\hline $\begin{array}{l}\text { Azotobacter } \\
\text { vinelandii }\end{array}$ & $3,0-4,5 \mu m$ & $\begin{array}{c}\mathrm{T}^{\circ}: 20-30^{\circ} \mathrm{C} \\
\mathrm{pH}: 7-8,5\end{array}$ & $\begin{array}{c}\text { Aminoácidos } \\
\text { Vitamina B2 y B3 } \\
\mathrm{CO}_{2} \\
\mathrm{~N}_{2} \\
\mathrm{NH}_{4} \\
\end{array}$ & $\begin{array}{c}\text { PHA } \\
\text { AG }\end{array}$ & $\begin{array}{l}\text { (Jiménez et al., } \\
\text { 2011) } \\
\text { (Chennappa et al., } \\
\text { 2019) }\end{array}$ \\
\hline $\begin{array}{l}\text { Azotobacter } \\
\text { beïjerinckii }\end{array}$ & $3,2-5,3 \mu \mathrm{m}$ & $\begin{array}{l}\mathrm{T}^{\circ}: 10-30^{\circ} \mathrm{C} \\
\mathrm{pH}: 4,9-5,5\end{array}$ & $\begin{array}{c}\text { Aminoácidos } \\
\text { Vitamina } \mathrm{B} 2 \text { y B3 } \\
\mathrm{CO}_{2} \\
\mathrm{NH}_{3} \\
\end{array}$ & $\begin{array}{c}\text { PHA } \\
\text { AG }\end{array}$ & $\begin{array}{l}\text { (Patil et al., 2020) } \\
\text { (Hindersah et al., } \\
\text { 2021) } \\
\text { (Blunt et al., 2018) }\end{array}$ \\
\hline $\begin{array}{l}\text { Azotobacter } \\
\text { paspali }\end{array}$ & $7,0-11,0 \mu \mathrm{m}$ & $\begin{array}{l}\mathrm{T}^{\circ}: 15-37^{\circ} \mathrm{C} \\
\mathrm{pH}: 6,0-7,5\end{array}$ & $\begin{array}{c}\text { Aminoácidos } \\
\text { Vitamina } \mathrm{B} 2 \text { y B3 } \\
\mathrm{CO}_{2} \\
\text { Ácido indolacético } \\
\end{array}$ & $\begin{array}{c}\text { PHA } \\
\text { AG }\end{array}$ & $\begin{array}{l}\text { (Patil et al., 2020) } \\
\text { (Saranraj \& } \\
\text { Sivasakthivelan, } \\
\text { 2017) }\end{array}$ \\
\hline $\begin{array}{l}\text { Azotobacter } \\
\text { armeniacus }\end{array}$ & $5,0-5,7 \mu m$ & $\begin{array}{c}T^{\circ}: 28-37^{\circ} \mathrm{C} \\
\mathrm{pH}: 6,0-7,5\end{array}$ & $\begin{array}{c}\text { Aminoácidos } \\
\text { Vitamina } \mathrm{B} 2 \text { y B3 } \\
\mathrm{CO}_{2} \\
\mathrm{NH}_{3} \\
\end{array}$ & $\begin{array}{c}\text { PHA } \\
\text { AG }\end{array}$ & (Patil et al., 2020) \\
\hline $\begin{array}{l}\text { Azotobacter } \\
\text { nigricans }\end{array}$ & $4,1-4,9 \mu \mathrm{m}$ & $\begin{array}{l}\mathrm{T}^{\circ}: 9-37^{\circ} \mathrm{C} \\
\mathrm{pH}: 6,0-7,5\end{array}$ & $\begin{array}{c}\text { Aminoácidos } \\
\text { Vitamina B2 y B3 } \\
\mathrm{CO}_{2} \\
\mathrm{NH}_{3}\end{array}$ & $\begin{array}{c}\text { PHA } \\
\text { AG }\end{array}$ & (Patil et al., 2020) \\
\hline $\begin{array}{l}\text { Azotobacter } \\
\text { salinestri }\end{array}$ & $2,0-4,0 \mu m$ & $\begin{array}{l}\mathrm{T}^{\circ}: 15-35^{\circ} \mathrm{C} \\
\mathrm{pH}: 6,0-7,5\end{array}$ & $\begin{array}{c}\text { Aminoácidos } \\
\text { Vitamina } \mathrm{B} 2 \text { y B3 } \\
\mathrm{CO}_{2} \\
\mathrm{NH}_{3}\end{array}$ & $\begin{array}{c}\text { PHA } \\
\text { AG }\end{array}$ & (Patil et al., 2020) \\
\hline
\end{tabular}<smiles>O=C[C@H](O)[C@H](O)[C@H](O)[C@H](O)C(=O)O</smiles><smiles>O=C[C@H](O)[C@H](O)[C@H](O)[C@H](O)C(=O)O</smiles>

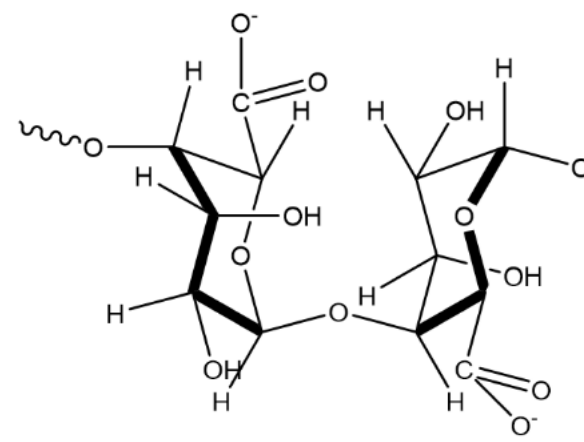

(c)

Figura 1. Estructura química del Alginato, (a) ácido manurónico (M); (b) ácido gulorónico (G); (c) estructura principal M y G.

El proceso de biosíntesis de este polímero se realiza bajo condiciones limitantes en la concentración de oxígeno disuelto en el medio, esto permite que la bacteria inicie un proceso de enquistamiento con la finalidad de proteger su membrana celular con una doble capa externa rica en AG (Pacheco-Leyva et al., 2016). El proceso de biosíntesis para obtener AG se inicia con la conversión de glucosa a fructosa-6-fosfato, la cual se isomeriza a manosa 6-P, luego es activada como GDP-manosa por la enzima GDPmanosa-pirofosforilasa. Después la enzima GDP-manosadeshidrogenasa a través de un proceso de oxidación convierte la GPD-manosa a GPD-manuronico, el cual es polimerizado y acetilado para formar la estructura química del alginato (Figura 2) (Campos \& Marti, 1996; Remminghorst \& Rehm, 2006; Galindo et al., 2007; Pacheco-Leyva et al., 2016). 
Si bien existen diferentes enzimas relacionadas con el proceso de biosíntesis de $A G$, son finalmente las enzimas GPD-manosa-deshidrogenasa y C-5 epimerasa las más importantes para la formación del polímero de AG, cuyos genes responsables para su codificación y producción se conocen como algD y algE respectivamente (Gawin et al., 2020; Hay et al., 2014).

Si bien ambas especies de Azotobacter producen AG, la presencia de bloques $\mathrm{G}$ y $\mathrm{M}$, el grado de polimerización y el peso molecular son características decisivas para establecer futuras aplicaciones industriales, las cuales se pueden controlar mediante la modificación de las condiciones del medio de cultivo tales como oxígeno disuelto, agitación, temperatura y pH (Galindo et al., 2007; Gawin et al., 2020). Por ejemplo, En A. vinelandii las bajas concentraciones de oxígeno disuelto ( $<5 \mathrm{mmol} / \mathrm{g} . \mathrm{h}$ ) permiten la obtención de AG de alto peso molecular (> 500 kDa) (Pacheco-Leyva et al., 2016); mientras que los pH menores a 5,5 en el medio de cultivo inhiben a la bacteria, consecuentemente la producción de AG se ve afectada (Clementi et al., 1995). Adicionalmente, los incrementos en la temperatura de 25 a $35^{\circ} \mathrm{C}$ favorecen la producción de AG en $~ 80 \%$ (Tabla 2).

Interesantemente, la modificación genética de estas especies de Azotobacter permite incrementar el peso molecular durante la biosíntesis de AG (Núñez et al., 2013). Por ejemplo, en A. vinelandii la inactivación de la actividad de la enzima alginato liasa combinado con una reducción en la concentración de oxígeno disuelto de 5 a $1 \%$ mejora la polimerización de $A G$, consecuentemente el peso molecular se incrementa significativamente en 1900 kDa en comparación con las cepas naturales (680 kDa) (Trujillo-Roldán et al., 2003). Por ello, diferentes trabajos se enfoquen en realizar modificaciones genéticas para mejorar su capacidad de sintetizar AG de alto peso molecular en comparación con las cepas naturales de Azotobacter (Tabla 3).

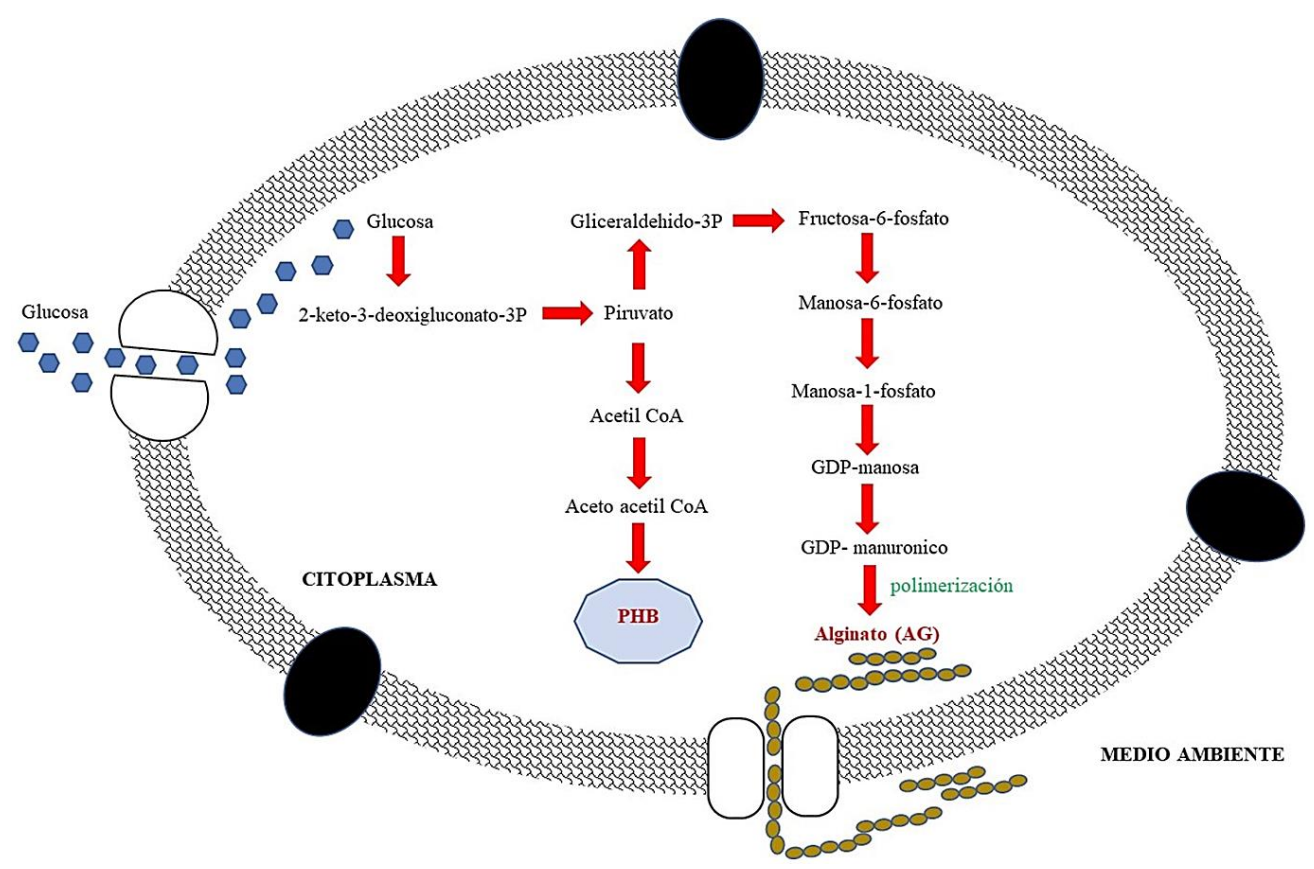

Figura 2. Diagrama del proceso de biosíntesis de Alginato y PHA.

Tabla 2

Parámetros óptimos de proceso para la obtención de AG a partir de especies de Azotobacter

\begin{tabular}{|c|c|c|c|c|c|c|}
\hline \multirow[t]{2}{*}{ Parámetro } & \multicolumn{2}{|c|}{ Oxígeno disuelto (mmol/g.h) } & \multicolumn{2}{|c|}{$\begin{array}{c}\text { Temperatura } \\
\left({ }^{\circ} \mathrm{C}\right)\end{array}$} & \multicolumn{2}{|c|}{$\mathrm{pH}$} \\
\hline & $1-5$ & $>5-10$ & $25-35$ & $>35-42$ & $5-6,0$ & $6,5-7,5$ \\
\hline Peso molecular (kDa) & $500-1350$ & $480-870$ & & & & \\
\hline Rendimiento $(\mathrm{g} / \mathrm{L})$ & & & $0,8-1,8$ & $<0,5$ & & \\
\hline Rendimiento $(\mathrm{g} / \mathrm{L})$ & & & & & $0,0-2,0$ & $>2,0-5,0$ \\
\hline
\end{tabular}

Adaptado desde Pacheco-Leyva et al. (2016) y Clementi et al. (1995)

Tabla 3

Producción de alginato con diferentes cepas modificadas de Azotobacter vinelandii a $29 \pm 1{ }^{\circ} \mathrm{C}$

\begin{tabular}{lccc}
\hline \multirow{2}{*}{ Identificación de la cepa modificada genéticamente } & Peso molecular & \multicolumn{2}{c}{ Condiciones } \\
\cline { 2 - 4 } & $(\mathrm{kDa})$ & $200-700$ & Oxígeno disuelto \% \\
\hline ATC9046 & $1900-770$ & $200-300$ & $1-5$ \\
CN26 & $1130-400$ & $200-300$ & 3 \\
DM & $1700-4000$ & $200-500$ & $1-5$ \\
Cepas no modificadas de Azotobacter & $480-870$ & \\
\hline
\end{tabular}

Adaptado desde Trujillo-Roldán et al. (2003); Peña et al. (2002); Flores et al. (2013); Pacheco-Leyva et al. (2016) 


\subsection{Síntesis y producción de polihidroxialcanoatos (PHA)} los polihidroxialcanoatos (PHA) comprenden una familia de poliésteres biodegradables, los cuales son producidos no solo por las especies del género Azotobacter, sino también por una amplia variedad de microorganismos (Chen et al., 2011). Si bien la estructura química de los PHAs está definida en función de la sustitución de su cadena lateral R (Figura 3), también es posible clasificarlos en función de la longitud de su cadena como PHA de cadena corta (consta de 3-5 monómeros de carbono) y PHA de cadena larga (consta de 6-14 monómeros de carbono) (Li et al., 2016).

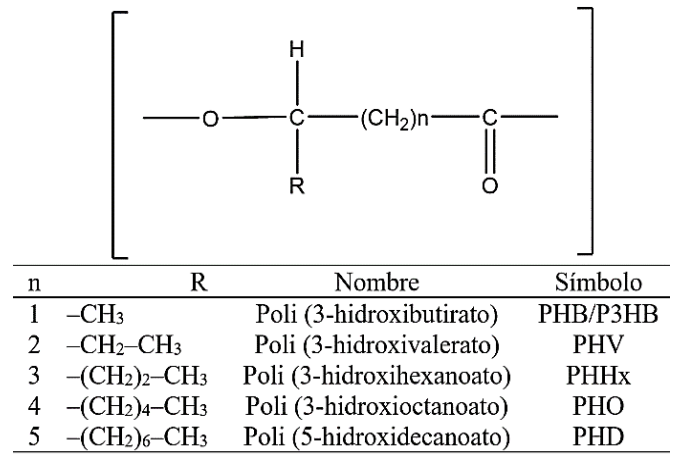

Figure 3. Estructura química de PHA.

El proceso de biosíntesis de los polímeros de PHA inicia bajo condiciones limitantes de nutrientes y condiciones adversas en el medio de crecimiento (El-Nahrawy et al., 2018), cuyo proceso se inicia con la conversión de glucosa a piruvato y acetil-CoA para formar acetoacetil-CoA. Luego, la enzima acetoacetil-CoA reductasa convierte este compuesto en 3-hidroxibutiril-CoA, y una polimerasa permite iniciar la polimerización del PHA (Figura 2). El gen responsable de la síntesis de la molécula de ácido polihidroxibutirico es reconocido como phbBAC, mientras que los genes phBP y phBR permiten la polimerización de gránulos de para formar diferentes formas de PHAs (Chen et al., 2011; Fa et al., 2001; Segura et al., 2003).

El poli-3-hidroxibutirato (PHB) y el polihidroxivalerato (PHV) son probablemente los tipos más comunes de PHAs producidos de forma intracelular por ambas especies de A. vinelandii y A. chroococcum (Narendran et al., 2018; Pozo et al., 2002; Yoneyama et al., 2015). La producción de ambos polímeros, puede ser regulada en función de las condiciones del medio de cultivo (Domínguez-Díaz et al., 2015a; El-Nahrawy et al., 2018). Por ejemplo, un incremento en la temperatura de 20 a $30^{\circ} \mathrm{C}$ mejora en $\sim 58 \%$ el rendimiento en la producción de PHB, mientras que las bajas concentraciones de oxígeno disuelto $(<5$ $\mathrm{mmol} / \mathrm{g} . \mathrm{h}$ ) permiten la obtención de PHB de alto peso molecular ( 1600 kDa). Además, es importante considerar que los valores cercanos a pH: 7 mejoran los rendimientos en la producción de este polímero (Tabla 4).

Si bien el peso molecular de ambos polímeros de PHB y PHV varían considerablemente en función de las condiciones de proceso, son finalmente los polímeros de alto peso molecular los más atractivos para la industria, las cuales se pueden conseguir mediante las modificaciones genéticas de las especies de Azotobacter (Mok et al., 2017). Por ejemplo, el peso molecular del PHB producido por las cepas naturales de Azotobacter varía entre 190 - 1600 kDa, mientras que las modificaciones genéticas de estas especies permiten que los polímeros de PHB presenten altos pesos moleculares (> 2000 kDa) (He \& Benson, 2016). Por ello, la modificación genética mediante el bloqueo de los genes pts $N$ y rsmA (genes reguladores negativos para la producción de PHB) ha permitido que las cepas modificadas de Azotobacter puedan producir polímeros de alto peso molecular (Domínguez-Díaz et al., 2015).

Otro aspecto importante a considerar es que las especies de Azotobacter pueden sintetizar simultáneamente ambos polímeros de PHAs y AG (Yoneyama et al., 2015). Por ejemplo, a $30^{\circ} \mathrm{C}$ y bajas concentraciones de oxígeno (< $5 \mathrm{mmol} / \mathrm{g}$.h) la producción de PHB es $\sim 2$ veces mayor comparada con la producción de AG (Mejía et al., 2010). Sin embargo, el solo hecho de producir ambos compuestos conlleva a un complejo proceso de purificación con la finalidad de separar ambos polímeros de PHB y AG. Por ello, diversos trabajos de investigación han demostrado que es posible bloquear los genes responsables de la producción de alginato con la finalidad de promover solo la producción de PHB o viceversa (Castillo et al., 2013; Flores et al., 2013).

\section{Usos y aplicaciones tecnológicas}

Si bien ambos biopolímeros de AG y PHAs son producidos simultáneamente por las especies de Azotobacter, la modificación genética puede permitir priorizar la producción de un compuesto en particular con la finalidad de que el proceso de extracción, purificación y comercialización sea económicamente atractivo (Jong-il Choi \& Lee 1999). Entonces, según sus propiedades físicas, químicas y térmicas ambos polímeros pueden ser utilizados en sectores de la industria alimentaria, farmacéutica, médica y agronómica.

Tabla 4

Parámetros óptimos de proceso para la obtención de PHB a partir de especies de Azotobacter

\begin{tabular}{|c|c|c|c|c|c|c|}
\hline \multirow{2}{*}{ Parámetro } & \multicolumn{2}{|c|}{ Oxígeno disuelto (mmol/g.h) } & \multicolumn{2}{|c|}{$\begin{array}{l}\text { Temperatura } \\
\left({ }^{\circ} \mathrm{C}\right) \\
\end{array}$} & \multicolumn{2}{|l|}{$\mathrm{pH}$} \\
\hline & $<5$ & $>5-10$ & $20-30$ & $>35^{\circ} \mathrm{C}$ & $1-5,0$ & $6,0-7,0$ \\
\hline Peso molecular (kDa) & 1600 & $190-550$ & & & & \\
\hline Rendimiento (g/L) & & & $2,4-3,8$ & $<0,2$ & & \\
\hline Rendimiento (g/L) & & & & & $0,3-1,6$ & $2,3-3,6$ \\
\hline
\end{tabular}

Adaptado desde Domínguez-Díaz et al. (2015); El-Nahrawy et al. (2018) 
Tabla 5

Alginato y sus potenciales aplicaciones tecnológicas

\begin{tabular}{|c|c|c|}
\hline Descripción & Características & Referencias \\
\hline \multicolumn{3}{|l|}{ Sector alimentario } \\
\hline Alginato & Forma geles termoestables. & (Smith \& Hong-Shum, 2011) \\
\hline Alginato de sodio & Forma geles estables de alta viscosidad. & (Larsen et al., 2015) \\
\hline Alginato de sodio - $\mathrm{Ca}^{2+}$ & Forma geles que permiten atrapar físicamente moléculas de agua. & (Qin et al., 2020) \\
\hline Alginato - glicerol & Películas biodegradables con alta flexibilidad y resistencia al agua. & (Parreidt et al., 2018) \\
\hline $\begin{array}{l}\text { Alginato de sodio - } \\
\text { pigmentos (bixina) }\end{array}$ & $\begin{array}{l}\text { Películas biodegradables resistentes con alta capacidad antioxidante } \\
\text { para reducir la oxidación de aceites. }\end{array}$ & (de Farias et al., 2020) \\
\hline $\begin{array}{l}\text { Alginato-almidón-PVOH - } \\
\text { aceite esencial }\end{array}$ & $\begin{array}{l}\text { Películas biodegradables activas, para prologar la vida útil de lechugas } \\
\text { frescas. }\end{array}$ & (Brandelero et al., 2016) \\
\hline \multicolumn{3}{|l|}{ Sector farmacéutico } \\
\hline Alginato - $\mathrm{Ca}^{2+}$ & $\begin{array}{l}\text { Liberación controlada de drogas químicas hidrofílicas, mediante la } \\
\text { formación de geles nanoporosos ( } ~ 5 \mathrm{~nm}) \text {. }\end{array}$ & (Lee et al., 2016) \\
\hline Alginato - Quitosano & $\begin{array}{l}\text { Nano encapsulación para la liberación controlada de cucurmina, un } \\
\text { compuesto con propiedades antiinflamatorias. }\end{array}$ & $\begin{array}{l}\text { (Hernandez-Patlan et al., } \\
\text { 2019) }\end{array}$ \\
\hline $\begin{array}{l}\text { Alinato - PLGA-PEG- } \\
\text { Quitosano }\end{array}$ & $\begin{array}{l}\text { Formación de geles rígidos de alta porosidad, biocompatibilidad, } \\
\text { adhesión y excelente mineralización para la regeneración de huesos. }\end{array}$ & (Venkatesan et al., 2015) \\
\hline Alginato de sodio y calcio & $\begin{array}{l}\text { Formación de apósitos para absorber el exceso de líquido de la herida, } \\
\text { mantener un ambiente fisiológicamente húmedo y minimizar las } \\
\text { infecciones bacterianas en el sitio de la herida. }\end{array}$ & $\begin{array}{l}\text { (Aderibigbe \& Buyana, } \\
\text { 2018) }\end{array}$ \\
\hline Alginato & $\begin{array}{l}\text { Regeneración de tejidos: vasos sanguíneos, proporcionar una liberación } \\
\text { sostenida y localizada de heparina. }\end{array}$ & (Kim et al., 2016) \\
\hline $\begin{array}{l}\text { Alginato/ } \\
\text { Ácido poliláctico }\end{array}$ & $\begin{array}{l}\text { Estudios in vitro en células mesenquimales del hueso en roedores } \\
\text { demostraron que pueden proliferarse en las estructuras diseñas con AG. }\end{array}$ & (Hu et al., 2016) \\
\hline \multicolumn{3}{|l|}{ Sector agricultura } \\
\hline $\begin{array}{l}\text { Alginato - lignosulfonato - } \\
\text { harinas }\end{array}$ & $\begin{array}{l}\text { Formación de hidrogeles para tratamiento del suelo fértil mediante la } \\
\text { liberación controlada de agua y nutrientes. }\end{array}$ & (Song et al., 2020) \\
\hline Alginato - poliacrilamida & $\begin{array}{l}\text { Formación de hidrogeles para una liberación controlada de fertilizantes } \\
\text { y nutrientes. }\end{array}$ & (Al Rohily et al., 2021) \\
\hline
\end{tabular}

\subsection{Alginatos}

EL AG, un polímero de bajo costo, de naturaleza inerte, biocompatible, biodegradable y e fácil gelación ha despertado el interés de la industria alimentaria, farmacéutica y agricultura (Puscaselu et al., 2020). En particular, Las especies de Azotobacter producen AG acetilado de alto peso molecular, por ello tienen una mayor preferencia como estabilizante y gelificante en la industria alimentaria (Furth et al., 2007). No obstante, este polímero en particular no solo puede ser usado como ingrediente alimentario, sino también puede ser destinado como aditivos en el sector médico debido a su biocompatibilidad, baja toxicidad y bajo costo (Gregory et al., 2012), así como en la mejora de la productividad de diferentes cultivos desde un punto de vista agronómico (Tabla 5).

\subsection{Polihidroxialcanoatos (PHA)}

Si bien el PHB y PHV pueden usarse en la fabricación de plásticos biodegradables para reemplazar al plástico sintético debido a su alta biodegradabilidad, así como a sus propiedades físicas y mecánicas; también estos polímeros pueden ser usados en la fabricación de dispositivos médicos debido a su biocompatibilidad y alta resistencia a temperaturas de esterilización. Adicionalmente, desde un punto de vista agronómico, la biodegradación de estos polímeros permite no solo mejorar la flora microbiana aerobia benéfica para los cultivos sino también permiten regular el contenido de $\mathrm{N}_{2}$ en el suelo (Tabla 6).

\section{Perspectivas y desafíos}

Si bien los polímeros de PHA y AG son producidos por las especies Azotobacter, las características físicas, químicas y mecánicas de cada polímero les confiere usos particulares en el sector alimentario, farmacéutico, médico y agronómico; esto debido a sus propiedades de biocompatibilidad, biodegradación y no toxicidad que ha permitido el desarrollo de muchos trabajos de investigación enfocados en el aprovechamiento de estos polímeros.

No obstante, durante la producción de PHAs se producen diferentes polímeros de manera simultánea entre ellos el PHB y PHV con características química y usos diferentes. Por lo tanto, es importante que desde un punto de vista de biotecnología se desarrollen nuevos estudios que permitan modificar genéticamente estas especies para la producción de un polímero en particular, con la finalidad no solo de reducir los costos de producción, sino también poder establecer nuevos parámetros de producción de estos polímeros. 
Tabla 6

PHA y sus potenciales aplicaciones tecnológicas

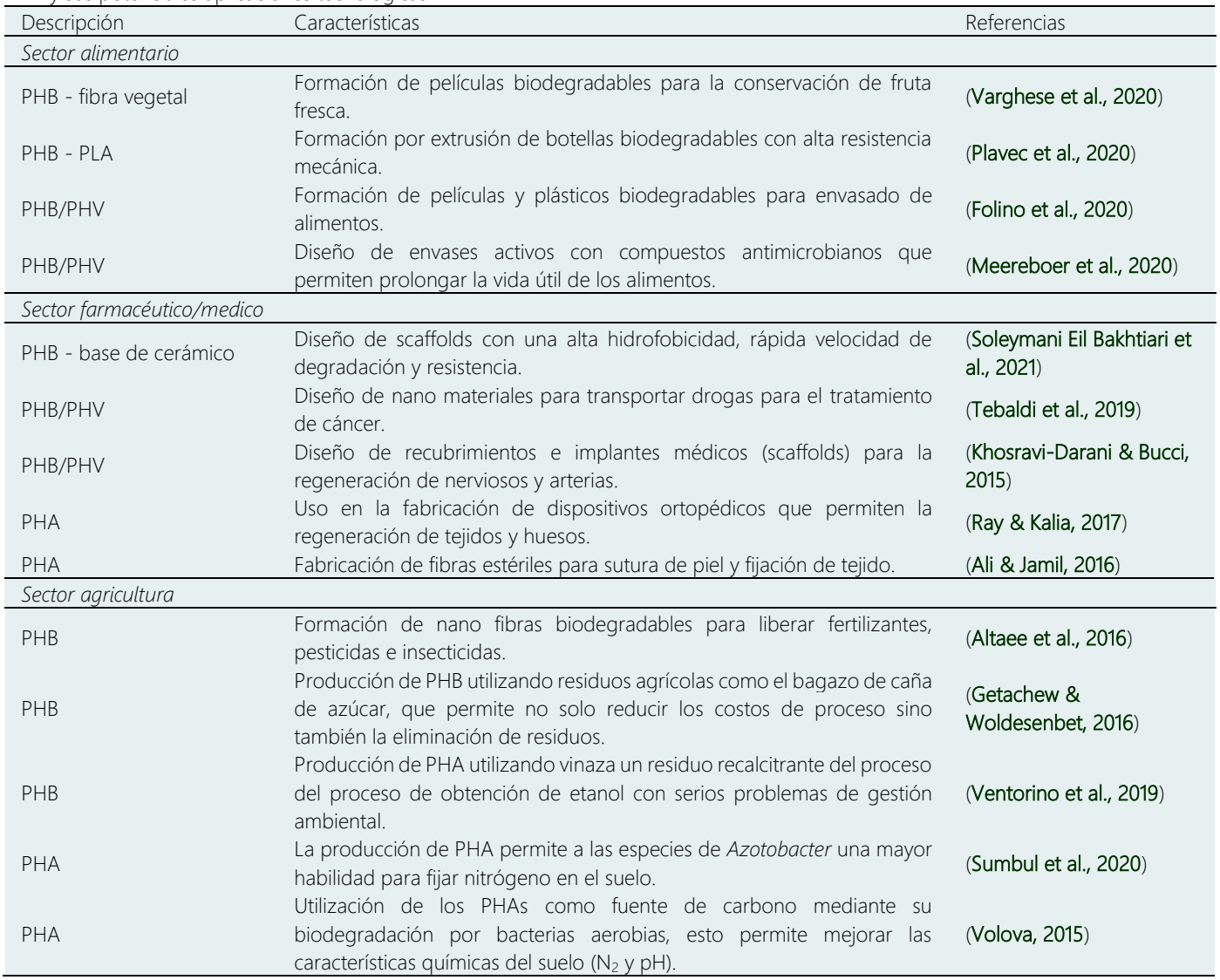

Con respecto a la producción de $\mathrm{AG}$ de fuentes bacterianas como las especies de Azotobacter, este proceso puede ser relativamente costoso sino se maneja a nivel industrial mediante el uso de reactores semicontinuos que controlen las condiciones del medio de cultivo. Por ello, es deseable que las futuras investigaciones se enfoquen en la modificación genética de estas especies con la finalidad de permitir que bajo condiciones normales de crecimiento puedan metabolizar estos polímeros.

Sin lugar a duda, el uso de AG y PHAs en el sector industrial puede llegar a tener diversas ventajas no solo desde un punto de vista económico sino también desde un punto de vista de cuidado del medio ambiente. Por lo tanto, el campo de desarrollo e investigación en ese sector siempre será atractivo debido al constante crecimiento de los sectores alimentario, farmacéutico, médico y agronómico.

\section{Conclusiones}

Los polímeros de alginato y PHAs son producidos por las especies de Azotobacter, cuyos genes responsables de producir estos polímeros son algD y phbBAC, respectivamente. La modificación genética permite la producción de un polímero de interés disminuyendo significativamente los costos de producción. Las características particulares de ambos polímeros permiten potenciales aplicaciones en la industria debido a su resistencia a la deformación, viscoelasticidad y propiedades de gelificación. Además, estos polímeros presentan una alta biocompatibilidad y biodegradabilidad para futuras aplicaciones en el sector médico. Adicionalmente, ambos polímeros tienen una rápida velocidad de biodegradación a condiciones atmosféricas normales, consecuentemente son amigables con el medio ambiente.

\section{ORCID}

N. L. Huamán-castilla (D) https://orcid.org/0000-0002-3748-0883

E. E. Allcca-Alca: (D) https://orcid.org/0000-0002-6116-7651

G. Allcca-Alca: (D) https://orcid.org/0000-0003-1397-9679

M. L. Quispe-Pérez: (D) https://orcid.org/0000-0002-8661-3806

\section{Referencias bibliográficas}

Aasfar, A., Bargaz, A., Yaakoubi, K., Hilali, A., Bennis, I., Zeroual, Y., \& Meftah Kadmiri, I. (2021). Nitrogen Fixing Azotobacter Species as Potential Soil Biological Enhancers for Crop Nutrition and Yield Stability. Frontiers in Microbiology, 12, 1-19.

Aderibigbe, B. A., \& Buyana, B. (2018). Alginate in wound dressings. Pharmaceutics, 10(2), 1-19.

Ahmadi, M., Madrakian, T., \& Ghavami, S. (2020). Preparation and Characterization of Simvastatin Nanocapsules: Encapsulation of Hydrophobic Drugs in Calcium Alginate. Methods in Molecular Biology, 2725, 47-56.

Al Rohily, K., El-Hamshary, H., Ghoneim, A., \& Modaihsh, A. (2021). Controlled release of phosphorus from superabsorbent phosphate-bound alginate-graft-polyacrylamide: Resistance to soil cations and release mechanism. ACS Omega, 5(51), 32919-32929.

Ali, I., \& Jamil, N. (2016). Polyhydroxyalkanoates: Current applications in the medical field. Frontiers in Biology, 11(1), 19-27.

Alshehrei, F. (2017). Biodegradation of Synthetic and Natural Plastic by Microorganisms. Journal of Applied \& Environmental Microbiology, 5(1), 8-19. 
Altaee, N., El-Hiti, G. A., Fahdil, A., Sudesh, K., \& Yousif, E. (2016). Biodegradation of different formulations of polyhydroxybutyrate films in soil. SpringerPlus, 5(1), 2-12.

Andrady, A. L., \& Neal, M. A. (2009). Applications and societal benefits of plastics. Philosophical Transactions of the Royal Society B: Biological Sciences, 364(1526), 1977-1984.

Gregory, S. K. (2012). Hydrogels and Their Mechanical Properties. In: Engineering Biomaterials for Regenerative Medicine: Novel Technologies for Clinical Applications. (S. K. Bhatia (ed.); $1^{\text {a }}$ ed.). Springer. New York. Pp. 127-140.

Blunt, W., Sparling, R., Gapes, D. J., Levin, D. B., \& Cicek, N. (2018). The role of dissolved oxygen content as a modulator of microbial polyhydroxyalkanoate synthesis. World Journal of Microbiology and Biotechnology, 34(8), 1-12

Brandelero, R. P. H., Brandelero, E. M., \& Almeida, F. M. de. (2016). Biodegradable films of starch/PVOH/alginate in packaging systems for minimally processed lettuce (Lactuca sativa L.). Ciência e Agrotecnologia, 40(5), 510-521.

Campos, A., \& Marti, J. M. (1996). Characterization of the gene coding for GDP-mannose dehydrogenase ( $\mathrm{algD}$ ) from Azotobacter vinelandii. $J$ Bacteriol, 178(7), 1793-1799.

Castillo, T., Heinzle, E., Peifer, S., Schneider, K., \& Peña M, C. F. (2013). Oxygen supply strongly influences metabolic fluxes, the production of poly(3hydroxybutyrate) and alginate, and the degree of acetylation of alginate in Azotobacter vinelandii. Process Biochemistry, 48(7), 995-1003.

Chamas, A., Moon, H., Zheng, J., Qiu, Y., Tabassum, T., et al. (2020). Degradation Rates of Plastics in the Environment. ACS Sustainable Chemistry and Engineering, 8(9), 3494-3511.

Chen, G. Q., Wu, Q., Jung, Y. K., \& Lee, S. Y. (2011). PHA/PHB. In: Comprehensive Biotechnology (M. Moo-Young (ed.) $2^{\text {a }}$ ed.). Elsevier B.V. Massachusetts. Pp. 217-227.

Chennappa, G., Udaykumar, N., Vidya, M., Nagaraja, H., Amaresh, Y. S., \& Sreenivasa, M. Y. (2019). Azotobacter-a natural resource for bioremediation of toxic pesticides in soil ecosystems. In: New and Future Developments in Microbial Biotechnology and Bioengineering: Microbial Biotechnology in Agro-environmental Sustainability (J. S. Singh and D.P. Singh (eds.) $1^{\text {a }}$ ed.). Elsevier B.V. Pp. 267-279.

Choi, J., \& Lee, S. Y. (1999). Factors affecting the economics of polyhydroxyalkanoate production by bacterial fermentation. Applied Microbiology and Biotechnology, 57(1), 13-21.

Clementi, F., Fantozzi, P., Mancini, F., \& Moresi, M. (1995). Optimal conditions for alginate production by Azotobacter vinelandii. Enzyme and Microbial Technology, 17(11), 983-988.

de Farias, Y. B., Coutinho, A. K., Assis, R. Q., \& Rios, A. de O. (2020). Biodegradable sodium alginate films incorporated with norbixin salts. Journal of Food Process Engineering, 43(2), 1-11.

Domínguez-Díaz, M., Meneses-Acosta, A., Romo-Uribe, A., Peña, C., Segura, D., \& Espin, G. (2015). Thermo-mechanical properties, microstructure and biocompatibility in poly- $\beta$-hydroxybutyrates (PHB) produced by OP and OPN strains of Azotobacter vinelandii. European Polymer Journal, 63, 101-112.

El-Nahrawy, S., Abd El-Kodoos, R. Y., Belal, E.-S. B., \& El-Shouny, W. (2018), Production of poly- $\beta$-hydroxybutyrate (PHB) by Azospirillum and Rhizobium sp. Environment, Biodiversity and Soil Security, 2(2018), 1-25.

Fa, S., Silberschmidt, D., Rehm, B., Pettinari, M. J., Va, G. J., Me, B. S., Qul, D. De, \& Mu, D.-. (2001). Poly (3-Hydroxybutyrate) Synthesis Genes in Azotobacter sp . Appl Environ Microbiol, 67(11), 5331-5334.

Fertah, M., Belfkira, A., Dahmane, E. montassir, Taourirte, M., \& Brouillette, F. (2017). Extraction and characterization of sodium alginate from Moroccan Laminaria digitata brown seaweed. Arabian Journal of Chemistry, 10, S3707-S3714.

Flores-Céspedes, F., Villafranca-Sánchez, M., \& Fernández-Pérez, M. (2020). Alginate-based hydrogels modified with olive pomace and lignin to removal organic pollutants from aqueous solutions. International Journal of Biological Macromolecules, 153, 883-891.

Flores, C., Moreno, S., Espín, G., Peña, C., \& Galindo, E. (2013). Expression of alginases and alginate polymerase genes in response to oxygen, and their relationship with the alginate molecular weight in Azotobacter vinelandii. Enzyme and Microbial Technology, 53(2), 85-91.

Folino, A., Karageorgiou, A., Calabrò, P. S., \& Komilis, D. (2020). Biodegradation of wasted bioplastics in natural and industrial environments: A review. Sustainability (Switzerland), 12(15), 1-37.

Francesca, C. (1997). Alginate Production by Azotobacter vinelandii. Critical Reviews in Biotechnology, 17(4), 327-361.

Furth, M. E., Atala, A., \& Van Dyke, M. E. (2007). Smart biomaterials design for tissue engineering and regenerative medicine. Biomaterials, 28(34), 5068-5073.

Galindo, E., Peña, C., Núñez, C., Segura, D., \& Espín, G. (2007). Molecular and bioengineering strategies to improve alginate and polydydroxyalkanoate production by Azotobacter vinelandii. Microbial Cell Factories, 6, 1-16
García, A., Segura, D., Espín, G., Galindo, E., Castillo, T., \& Peña, C. (2014a). High production of poly- $\beta$-hydroxybutyrate (PHB) by an Azotobacter vinelandii mutant altered in PHB regulation using a fed-batch fermentation process. Biochemical Engineering Journal, 82, 117-123.

García, A., Castillo, T., Ramos, D., Ahumada-Manuel, C. L., Núñez, C., Galindo, E., Büchs, J., \& Peña, C. (2020). Molecular weight and viscosifying power of alginates produced by mutant strains of Azotobacter vinelandii under microaerophilic conditions. Biotechnology Reports, 26, 1-12.

Gauri, S. S., Mandal, S. M., \& Pati, B. R. (2012). Impact of Azotobacter exopolysaccharides on sustainable agriculture. Applied Microbiology and Biotechnology, 95(2), 331-338

Gautam, R., Bassi, A. S., \& Yanful, E. K. (2007). A Review of Biodegradation of Synthetic Plastic and Foams. Applied Biochemistry and Biotechnology, 147(109), 315-323

Gawin, A., Tietze, L., Aarstad, O. A., Aachmann, F. L., Brautaset, T., \& Ertesvåg, H. (2020). Functional characterization of three Azotobacter chroococcum alginate-modifying enzymes related to the Azotobacter vinelandii AlgE mannuronan C-5-epimerase family. Scientific Reports, 10(1), 1-14.

Getachew, A., \& Woldesenbet, F. (2016). Production of biodegradable plastic by polyhydroxybutyrate (PHB) accumulating bacteria using low cost agricultural waste material. BMC Research Notes, 9(1), 1-9.

Geyer, R., Jambeck, J. R., \& Law, K. L. (2017). Production, use, and fate of all plastics ever made. Science Advances, 3(7), 25-29.

Hassett, D. J., \& Hassett, D. J. (1996). Anaerobic Production of Alginate by Pesudomonas aeruginosa. Microbiology, 178(24), 7322-7325.

Hay, I. D., Wang, Y., Moradali, M. F., Rehman, Z. U., \& Rehm, B. H. A. (2014) Genetics and regulation of bacterial alginate production. Environmental Microbiology, 16(10), 2997-3011.

He, W., \& Benson, R. (2016). Polymeric Biomaterials. In: Applied Plastics Engineering Handbook Processing, Materials, and Applications (M. Kutz (ed.) $2^{a}$ ed.). Elsevier B.V. Massachusetts. Pp. 145-164

Hernandez-Patlan, D., Solis-Cruz, B., Cano-Vega, M. A., Beyssac, E., Garrait, G., Hernandez-Velasco, X., Lopez-Arellano, R., Tellez, G., \& RiveraRodriguez, G. R. (2019). Development of Chitosan and Alginate Nanocapsules to Increase the Solubility, Permeability and Stability of Curcumin. Journal of Pharmaceutical Innovation, 14(2), 132-140.

Hindersah, R., Kamaluddin, N. N., Samanta, S., Banerjee, S., \& Sarkar, S. (2021). Role and perspective of Azotobacter in crops production. Sains Tanah, 17(2), 170-179

Hu, Y., Ma, S., Yang, Z., Zhou, W., Du, Z., Huang, J., Yi, H., \& Wang, C. (2016). Facile fabrication of poly(L-lactic acid) microsphere-incorporated calcium alginate/hydroxyapatite porous scaffolds based on Pickering emulsion templates. Colloids and Surfaces B: Biointerfaces, 140, 382-291.

Jiménez, D. J., Montaña, J. S., \& Martínez, M. (2011). Characterization of free nitrogen fixing bacteria of the genus Azotobacter in organic vegetablegrown colombian soils. Brazilian Journal of Microbiology, 42, 846-858.

Khosravi-Darani, K. \& Bucci, D. Z. (2015). Application of poly(hydroxyalkanoate) in food packaging: Improvements by nanotechnology. Chemical and Biochemical Engineering Quarterly, 29(2), 275-285.

Kim, H., Park, H., Lee, J. W., \& Lee, K. Y. (2016). Magnetic field-responsive release of transforming growth factor beta 1 from heparin-modified alginate ferrogels. Carbohydrate Polymers, 151, 467-473.

Kong, H. (2000). Isolation and characterization of alginate from hong kong brown seaweed. an evaluation of the potential use of the extracted alginate as food ingredient. University of Hong Kong. China.

Larsen, B. E., Bjørnstad, J., Pettersen, E. O., Tønnesen, H. H., \& Melvik, J. E. (2015). Rheological characterization of an injectable alginate gel system. BMC Biotechnology, 15(1), 1-12.

Lee, K. Y., \& Mooney, D. J. (2012). Alginate: Properties and biomedical applications. Progress in Polymer Science (Oxford), 37(1), 106-126.

Lee, S., Kim, Y.-C., \& Park, J.-H. (2016). Zein-alginate based oral drug delivery systems: Protection and release of therapeutic proteins. International Journal of Pharmaceutics, 575(1-2), 300-306

Lenart, A. (2012). Occurrence, characteristics, and genetic diversity of Azotobacter chroococcum in various soils of Southern Poland. Polish Journal of Environmental Studies, 21(2), 415-424

Li, Z., Yang, J., \& Loh, X. J. (2016). Polyhydroxyalkanoates: Opening doors for a sustainable future. NPG Asia Materials, 8(4), 1-20.

Lorbeer, A. J., Charoensiddhi, S., Lahnstein, J., Lars, C., Franco, C. M. M., Bulone, V., \& Zhang, W. (2017). Sequential extraction and characterization of fucoidans and alginates from Ecklonia radiata, Macrocystis pyrifera, Durvillaea potatorum, and Seirococcus axillaris. Journal of Applied Phycology, 29(3), 1515-1526.

Martyniuk, S., \& Martyniuk, M. (2003). Occurrence of Azotobacter spp. in some Polish soils. Polish Journal of Environmental Studies, 12(3), 371374.

Meereboer, K. W., Misra, M., \& Mohanty, A. K. (2020). Review of recent advances in the biodegradability of polyhydroxyalkanoate (PHA) bioplastics and their composites. Green Chemistry, 22(17), 5519-5558. 
Mejía, M. Á., Segura, D., Espín, G., Galindo, E., \& Peña, C. (2010). Two-stage fermentation process for alginate production by Azotobacter vinelandii mutant altered in poly- $\beta$-hydroxybutyrate (PHB) synthesis. Journal of Applied Microbiology, 108(1), 55-61.

Mohapatra, S., Maity, S., Dash, H. R., Das, S., Pattnaik, S., Rath, C. C., \& Samantaray, D. (2017). Bacillus and biopolymer: Prospects and challenges. Biochemistry and Biophysics Reports, 12, 206-213.

Mok, P. S., Sudesh, K., Liew, P. W. Y., Jong, B. C., \& Najimudin, N. (2017). Characterisation of polyhydroxyalkanoate production by mutant Azotobacter vinelandii. Malaysian Applied Biology, 46(1), 93-100.

Nagarajan, A., \& Zackaria, A. (2016). Mini review on Alginate: Scope and Future perspectives. Journal of Algal Biomass Utilization, 7(1), 45-55.

Nair, N. R., Sekhar, V. C., Nampoothiri, K. M., \& Pandey, A. (2016). Biodegradation of Biopolymers. In: Current Developments in Biotechnology and Bioengineering: Production, Isolation and Purification of Industrial Products (A. Pandey, S. Negi, C. R. Soccol (eds); $1^{a}$ ed.). Elsevier B.V. Massachusetts. Pp. 739-755.

Narendran, R., Maleeka Begum, S. F., \& Ayyppadasan, G. (2018). Production and optimization of polyhydroxy butyrate (phb) from azotobacter chroococcum (a3) strain for scale up using central composite design. Asian Journal of Microbiology, Biotechnology \& Environmental Sciences, 20(4), 1294-1305.

Núñez, C., Peña, C., Kloeckner, W., Hernández-Eligio, A., Bogachev, A. V., Moreno, S., ... \& Espín, G. (2013). Alginate synthesis in Azotobacter vinelandii is increased by reducing the intracellular production of ubiquinone. Applied microbiology and biotechnology, 97(6), 2503-2512.

Owlia, P., Nosrati, R., Saderi, H., Olamaee, M., Rasooli, I., \& Akhavian Tehrani, A. (2012). Correlation between nitrogen fixation rate and alginate productivity of an indigenous Azotobacter vinelandii from Iran. Iranian Journal of Microbiology, 4(3), 153-159.

Pacheco-Leyva, I., Guevara Pezoa, F., \& Díaz-Barrera, A. (2016). Alginate biosynthesis in azotobacter vinelandii: Overview of molecular mechanisms in connection with the oxygen availability. International Journal of Polymer Science, 2016, 11-13.

Parreidt, T. S., Müller, K., \& Schmid, M. (2018). Alginate-based edible films and coatings for food packaging applications. Foods, 7(10), 1-38

Patil, S. V., Mohite, B. V., Patil, C. D., Koli, S. H., Borase, H. P., \& Patil, V. S. (2020). Azotobacter. In: Beneficial Microbes in Agro-Ecology (N. Amaresan, M. S. Kumar, K. Annapurna, K. Kumar, A. Sankaranarayanan (eds); $1^{\text {a }}$ ed.). Elsevier B.V. Massachusetts. Pp. 397-426.

Peña, C., Campos, N., \& Galindo, E. (1997). Changes in alginate molecwlar mass distributions, broth viscosity and morphology of Azotobacter vinelandii cultured in shake flasks. Applied Microbiology and Biotechnology, 48(4), 510-515

Peña, C., Miranda, L., Segura, D., Nunez, C., Espin, G., \& Galindo, E. (2002) Alginate production by Azotobacter vinelandii mutants altered in polybeta-hydroxybutyrate and alginate biosynthesis. Microbial Biotechnology, 29(2), 209-213.

Plackett, D., \& Siró, I. (2011). Polyhydroxyalkanoates (PHAs) for food packaging. In: Multifunctional and Nanoreinforced Polymers for Food Packaging (J. M. Lagarón (ed.); 1a ed.). Elsevier B.V. Massachusetts. Pp. 498-526.

Plavec, R., Hlaváčiková, S., Omaníková, L., Feranc, J., Vanovčanová, Z., Tomanová, K., Bočkaj, J., Kruželák, J., Medlenová, E., Gálisová, I., Danišová, L., Přikryl, R., Figalla, S., Melčová, V., \& Alexy, P. (2020). Recycling possibilities of bioplastics based on PLA/PHB blends. Polymer Testing, 92, 106880

Pozo, C., Martínez-Toledo, M. V., Rodelas, B., \& González-López, J. (2002) Effects of culture conditions on the production of polyhydroxyalkanoates by Azotobacter chroococcum $\mathrm{H} 23$ in media containing a high concentration of alpechín (wastewater from olive oil mills) as primary carbon source. Journal of Biotechnology, 97(2), 125-131.

Puscaselu, R. G., Lobiuc, A., Dimian, M., \& Covasa, M. (2020). Alginate: From food industry to biomedical applications and management of metabolic disorders. Polymers, 12(10), 1-30.

Qin, Y., Zhang, G., \& Chen, H. (2020). The applications of alginate in functional food products. Journal of Nutrition \& Food Sciences, 3(1), 1-9.

Qu, B., \& Luo, Y. (2020). Chitosan-based hydrogel beads: Preparations, modifications and applications in food and agriculture sectors - A review. International Journal of Biological Macromolecules, 152, 437-448.

Ray, S., \& Kalia, V. C. (2017). Biomedical Applications of Polyhydroxyalkanoates. Indian Journal of Microbiology, 57(3), 261-269.

Rehm, B. H. A. (2010). Bacterial polymers: biosynthesis, modifications and applications. Nature Reviews. Microbiology, 8(8), 578-592.
Remminghorst, U., \& Rehm, B. H. A. (2006). Alg44, a unique protein required for alginate biosynthesis in Pseudomonas aeruginosa. FEBS Letters, 580(16), 3883-3888

Rosato, D. V., Di Mattia, D. P., Rosato, D. V., Rosato, D. V., Di Mattia, D. P., \& Rosato, D. V. (1991). The Structure and Basic Properties of Plastics. In: Designing with Plastics and Composites: A Handbook (D. Rosato (ed.); $1^{a}$ ed.). Springer. Netherlands. Pp. 61-123.

Saranraj, P., \& Sivasakthivelan, P. (2017). Biological nitrogen fixation by azotobacter sp. - a review. Asian Journal of Multidisciplinary Research, 3(5), 1274-1284.

Savenkova, L., Gercberga, Z., Nikolaeva, V., Dzene, A., Bibers, I., \& Kalnin, M. (2000). Mechanical properties and biodegradation characteristics of PHB-based films. Process Biochemistry, 35(6), 573-579.

Scott, G. (2015). Polymers in Modern life. In: Polymers and the Environment

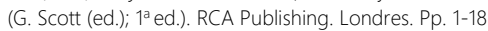

Segura, D., Cruz, T., \& Espín, G. (2003). Encystment and alkylresorcino production by Azotobacter vinelandii strains impaired in poly-betahydroxybutyrate synthesis. Archives of Microbiology, 179(6), 437-443.

Smith, J, \& Hong-Shum, L. (2011). Food Additives Data Book. Willey-Blackwell Science Ltd

Soleymani Eil Bakhtiari, S., Karbasi, S., \& Toloue, E. B. (2021). Modified poly(3hydroxybutyrate)-based scaffolds in tissue engineering applications: A review. International Journal of Biological Macromolecules, 166, 986-998.

Song, B., Liang, H., Sun, R., Peng, P., Jiang, Y., \& She, D. (2020). Hydrogel synthesis based on lignin/sodium alginate and application in agriculture. International Journal of Biological Macromolecules, 144, 219-230.

Sumbul, A., Ansari, R. A., Rizvi, R., \& Mahmood, I. (2020). Azotobacter: A potential bio-fertilizer for soil and plant health management. Saudi Journal of Biological Sciences, 27(12), 3634-3640. 4

Tebaldi, M. L., Maia, A. L. C., Poletto, F., de Andrade, F. V., \& Soares, D. C. F. (2019). Poly(-3-hydroxybutyrate-co-3-hydroxyvalerate) (PHBV): Current advances in synthesis methodologies, antitumor applications and biocompatibility. Journal of Drug Delivery Science and Technology, 57, 115-126.

Trujillo-Roldán, M., Moreno, S., Segura, D., Galindo, E., \& Espín, G. (2003). Alginate production by an Azotobacter vinelandii mutant unable to produce alginate lyase. Applied Microbiology and Biotechnology, 60(6), 733-737.

Urbanek, A. K., Rymowicz, W., \& Mirończuk, A. M. (2018). Degradation of plastics and plastic-degrading bacteria in cold marine habitats. Applied Microbiology and Biotechnology, 102(18), 7669-7678.

Urtuvia, V., Maturana, N., Acevedo, F., Peña, C., \& Díaz-Barrera, A. (2017) Bacterial alginate production: an overview of its biosynthesis and potential industrial production. World Journal of Microbiology and Biotechnology, 33(11).

Van De Velde, K., \& Kiekens, P. (2002). Biopolymers: Overview of several properties and consequences on their applications. Polymer Testing, 27(4), 433-442.

Varghese, S. A., Pulikkalparambil, H., Rangappa, S. M., Siengchin, S., \& Parameswaranpillai, J. (2020). Novel biodegradable polymer films based on poly(3-hydroxybutyrate-co-3-hydroxyvalerate) and Ceiba pentandra natural fibers for packaging applications. Food Packaging and Shelf Life, 25, 100538.

Venkatesan, J., Bhatnagar, I., Manivasagan, P., Kang, K. H., \& Kim, S. K. (2015) Alginate composites for bone tissue engineering: A review. International Journal of Biological Macromolecules, 72, 269-281.

Ventorino, V., Nicolaus, B., Di Donato, P., Pagliano, G., Poli, A., Robertiello, A. lavarone, V., \& Pepe, O. (2019). Bioprospecting of exopolysaccharideproducing bacteria from different natural ecosystems for biopolymer synthesis from vinasse. Chemical and Biological Technologies in Agriculture, 6(1), 1-9

Vieira, M. G. A., Da Silva, M. A., Dos Santos, L. O., \& Beppu, M. M. (2011). Natural-based plasticizers and biopolymer films: A review. European Polymer Journal, 47(3), 254-263.

Volova, T. G. (2015). Biodegradation of Polyhydroxyalkanoates in Natural Soils. Journal of Siberian Federal University. Biology, 8(2), 152-167.

Volova, T. G., Gladyshev, M. I., Trusova, M. Y., \& Zhila, N. O. (2010). Degradation of polyhydroxyalkanoates in eutrophic reservoir. Polymer Degradation and Stability, 64(July), 1294-1301.

Yoneyama, F., Yamamoto, M., Hashimoto, W., \& Murata, K. (2015). Production of polyhydroxybutyrate and alginate from glycerol by azotobacter vinelandii under nitrogen-free conditions. Bioengineered, 6(4), 209-217. 